\title{
It is Time for Action in the Struggle against Antibiotic-Resistance, Let's Start Reducing or Replacing Antibiotics in Agriculture
}

Maria Grazia Cusimano* and Domenico Schillaci

Department of Biological Chemical and Pharmaceutical Science and Technology, University of Palermo, Italy

\section{Introduction}

Million people around the world are infected each year and thousands of them die from pathogens that are resistant to the treatment by any of known antibiotics [1]. The excessive use and abuse of antibiotics in clinical setting and in agriculture, added to the great ability of microorganisms to evolve, are the causes of the wide spread of isolates resistant to all major classes of current antibiotics. It is time for action in order to tackle antimicrobial-resistance (AMR), which can be considered a major threat to global health care and security. While we are waiting for new antimicrobial molecules and strategies, shorter-term approaches are needed to address the menace of AMR and preserve the efficacy of current antibiotics.

For more than 70 years, the antibiotics have been used for the treatment of infectious diseases of food producing animals and to a lower level, antimicrobial substances have also been put on plant crops. The quantity of antibiotics used in animal husbandry - both terrestrial and aquatic - it is very high worldwide, percentages of antibiotics consumed in farms equal to $84 \%$ in China and $70 \%$ in USA, indicate that probably the amount of antibiotics used in agriculture at global level is superior to that for clinical use [2]. In industrialized farming, antimicrobials are sometimes used for disease prevention and in many countries; antimicrobials are added in sub-therapeutic concentrations to animal feed as growth-promoter. In North America the antibiotic use in agriculture is scarcely regulated, in Europe, stricter laws governing the use of antibiotics on farms and in 2006 it was finally prohibited their employment as a way to stimulate the growth. Unfortunately, we should consider that 110 countries do not have any law on the proper conditions for the import, production, distribution and use of veterinary products, including antimicrobial agents [3]. Nevertheless, since 2010, the World Organization for Animal Health (OIE), alongside the World Health Organization (WHO) and Food and Agriculture Organization of the United Nations (FAO), has been engaged in a "Tripartite Alliance" to contrast globally AMR in the short, medium and long terms. Such Tripartite alliance also controls the origin of medicines, obtain reliable data on imports, trace their circulation, and evaluate the quality of the products in circulation. In this way they created a global database for monitoring the use of antimicrobial agents and to combat AMR [4].

Humans and animals share many common pathogens and commensal bacteria and all microorganisms have a great ability to adapt, to evolve, and to survive by developing resistance to any known supplied antimicrobial. AMR from animals can be transferred to humans in two manners: i. the food-supply, in particular resistant strains of Campylobacter and Salmonella, two major food-borne pathogens, come from livestock; ii. Resistance genes from animal bacteria spread in the food and environment (by animal waste) could be transferred to human pathogens by horizontal gene transfer [5]. It has also been described a direct transmission from animals infected with Staphylococcus aureus methicillin resistant (MRSA) to farm workers [6].

In many cases, antimicrobials used in agriculture and in clinical setting are equal, that is a risk for the rise and spread of resistant bacteria, including common pathogens in both animals and humans. WHO recommends that some classes of antibiotics such as carbapenems, lipopeptides and oxazolidinones, drugs that do not have veterinary correspondent, should not be used in animal husbandry [7].

The OIE suggests a judicious use of antibiotics by trained professionals, such as veterinarians, to limit the spread of resistance from animals to humans, but such approach is not a solution that can stop in permanent way the antibiotic-resistance. Reducing the antibiotics and replacing them on farms with new treatments, will be needed. Plant extracts are being assessed as alternatives to antibiotics and some have antimicrobial activity, and other beneficial effects for animal and human health [8]. Moreover, plant-derived compounds have long been used to treat microbial infections and have gained much interest for the treatment of natural form of resistance such as biofilms [9]. One of these, curcumin, a major constituent of turmeric rhizomes (Curcuma longa), has been selected for its non-toxic nature. It also traditionally used for its benefit on human health because they contain a great number of secondary metabolites. Among those, curcuminoids have anti-inflammatory, hypoglicemic, anti-carcinogenic, anti-oxidative, antimicrobial and antifungal effects. Curcumin also exhibited potent inhibitory activity against $S$. aureus cell adhesion to fibronectin [10]. The interference with the adhesion, the first step of pathogenic process, can modulate virulence mechanisms like colonization, invasion of host tissue and biofilm formation. In our laboratory, we found that curcumin and a number of curcumin derivatives, were ineffective on planktonic forms of all tested strains (MIC greater than $100 \mu \mathrm{g} / \mathrm{ml}$ ) indicating an absence of effect on bacterial growth. However, most of tested compounds interfered with biofilm formation especially of $S$. aureus but also Pseudomonas aeruginosa and E.coli. The biological activity of such compounds is that typical of anti-virulence agents, because they do not target bacterial viability, therefore they should impose a low selection pressure rendering less likely the development of resistance. Curcumin and its derivatives, so as essential oils, spices, and various other plant extracts, could reduce or replace the use of antibiotics on the farms [8].

In addition to scientific research that should be adequately funded, another level of intervention in the preservation of life-saving molecules, such as antibiotics, involves more political and social aspects. For example, the responsible use of antibiotics, a reduction in antibiotic prescribing and finally the reduction on the use of such

*Corresponding author: Maria Grazia Cusimano, Department of Biological Chemical and Pharmaceutical Science and Technology, University of Palermo, Italy, Tel: +3909123891914; E-mail: mariagrazia.cusimano@unipa.it

Received November 18, 2016; Accepted December 20, 2016; Published December 27, 2016

Citation: Cusimano MG, Schillaci D (2016) It is Time for Action in the Struggle against Antibiotic-Resistance, Let's Start Reducing or Replacing Antibiotics in Agriculture. J Microb Biochem Technol 8: 507-508. doi: 10.4172/19485948.1000334

Copyright: (c) 2016 Cusimano MG, et al. This is an open-access article distributed under the terms of the Creative Commons Attribution License, which permits unrestricted use, distribution, and reproduction in any medium, provided the original author and source are credited. 
Citation: Cusimano MG, Schillaci D (2016) It is Time for Action in the Struggle against Antibiotic-Resistance, Let's Start Reducing or Replacing Antibiotics in Agriculture. J Microb Biochem Technol 8: 507-508. doi: 10.4172/1948-5948.1000334

molecules in the farms. Finally, the ban of third-line antibiotics that are often the last-resort molecules in the therapy against human pathogens, become resistant to the classes of the more common antibiotics, should be obliged in animal husbandry and in agriculture.

To focus on human and animal health together with the concern for the environment, the concept of "One Health" is more than appropriate if we want to address and resolve this complex problem [11].

\section{References}

1. Dodds DR (2016) Antibiotic resistance: a current epilogue. Biochem Pharmacol 2952: 30467-30471.

2. O'Neill J (2015) Antimicrobials in agriculture and the environment: reducing unnecessary use and waste the review on antimicrobial resistance, pp: 1-41.

3. Harbarth S, Balkhy HH, Goossens H, Jarlier V, Kluytmans J, et al. (2015) Antimicrobial resistance: one world, one fight! Antimicrobial Resistance and Infection Control 4: 1-15.

4. Nathan C, Cars O (2014) Antibiotic resistance-problems, progress and prospects. New Engl J Med 371: 1761-1763.
5. Chen Z, Jiang X (2014) Microbiological safety of chicken litter or chicken litterbased organic fertilizers: Review agriculture. 4: 1-29.

6. Carfora V, Giacinti G, Sagrafoli D, Marri N, Giangolini G, (2016) Methicillinresistant and methicillin-susceptible Staphylococcus aureus in dairy sheep and in-contact humans: An intra-farm study. J Dairy Sci 99: 4251-4258.

7. Bronson JJ, Barrett JF (2001) Quinolone, everninomycin, glycylcycline carbapenem, lipopeptide and cephem antibacterials in clinical development Curr Med Chem 8: 1775-1793.

8. Diaz-Sanchez S, D'Souza D, Biswas D, Hanning I (2015) Botanical alternative to antibiotics for use in organic poultry production. Poult Sci 94: 1419-1430.

9. Schillaci D, Vitale M (2012) Biofilm related to animal health, zoonosis and food transmitted diseases: Alternative targets for antimicrobial strategy. J Microb Biochem Technol, 4

10. Park BS, Kim JG, Kim MR, Lee SE, Takeoka GR, et al. (2005) Curcuma longa L. constituents inhibit sortase $A$ and Staphylococcus aureus cell adhesion to fibronectin. J Agric Food Chem 53: 9005-9009.

11. Robinson TP, Bu DP, Carrique-Mas J, Fèvre EM, Gilbert M, et al. (2016) Antibiotic resistance is the quintessential one health issue. Trans $\mathrm{R}$ Soc Trop Med Hyg 110: 377-380. 